eISSN: 2659-6482

DOI: https://doi.org/10.14201/pmrt.22154

\title{
CHANT DE TROUVÈRE, DE KURT SCHINDLER: ESTRENO Y PUBLICACIÓN EN NUEVA YORK
}

\author{
Kurt Schindler's Chant de Trouvère: premiere and publication \\ in New York
}

Claudia RAMÍREZ GARCÍA, Yolanda ALONSO VICARIO y

Doris Aracely GÁLVEZ MÉNDEZ

Universidad de Salamanca

idu004467@usal.es

RESUMEN: Este artículo propone un acercamiento a Chant de Trouvère (Troubadour song), canción medieval editada por Kurt Schindler en 1911 para la casa editorial G. Schirmer, a solo un año de su estreno por el Coro MacDowell en el hotel Waldorf-Astoria de Nueva York. Con el propósito de analizar el contexto en el que tuvo lugar su edición se abordan aspectos puntuales de la trayectoria biográfica de Kurt Schindler, se analizan las características musicales que distinguen a la partitura y, por último, se estudian las redes sociales que condicionaron su estreno.

Palabras clave: Kurt Schindler; Troubadour song; G. Schirmer; Coro MacDowell; redes.

ABSTRACT: This article proposes an approach to Chant de Trouvère (Troubadour song), a medieval song edited by Kurt Schindler in 1911 for the G. Schirmer publishing house, just one year after its premiere by the MacDowell Choir at the Waldorf-Astoria hotel in New York. In order to analyze the context in which its edition took place, specific aspects of Kurt Schindler's biographical trajectory are addressed, the musical characteristics that distinguish the score are analyzed and finally the social networks that conditioned its premiere are studied. 
C. RAMÍREZ GARCÍA; Y. ALONSO VICARIO Y D. A. GÁLVEZ MÉNDEZ

CHANT DE TROUVĖRE, DE KURT SCHINDLER: ESTRENO Y PUBLICACIÓN EN NUEVA YORK

Key words: Kurt Schindler; Troubadour song; G. Schirmer; MacDowell Choir; networks.

\section{INTRODUCCIÓN}

En el año 1911 la editorial de música G. Schirmer publicó Chant de Trowvère o Troubadour song, una canción con letra y música de Kurt Schindler, basada en una melodía del siglo XII, dedicada al amigo del autor M. Edmond Clément. Para esa fecha, dicha casa editorial, fundada en Nueva York en 1861 y que actualmente es considerada la más antigua en activo en Estados Unidos, ya era considerada una institución de prestigio. Kurt Schindler llevaba en ese momento pocos años residiendo en Norteamérica, ¿de qué manera logró Schindler que una casa editorial como la Schirmer apostara por su trabajo? ¿Por qué fue relevante para dicha casa editorial la publicación de Chant de Trouvère?

En este trabajo, estudiaremos la biografía de Kurt Schindler, sus primeros estudios musicales, su trayectoria profesional y cómo llegó a estar en lo más alto del selecto círculo burgués de Nueva York. Luego se hará un análisis de la obra que nos interesa, Chant de Tronvère. Como tercer apartado se hará un repaso al contexto en el que se efectuó su estreno, los artistas que actuaron y las personas que patrocinaban el concierto. Para terminar, las conclusiones.

\section{APUNTES PARA UNA BIOGRAFÍA DE KURT SCHINDLER}

Las características de nuestro protagonista, el músico Kurt Schindler, hacen realmente complicado el abordaje de un estudio biográfico. El muchas veces intencionado misterio de sus ocupaciones y viajes nos dificulta en gran medida este cometido. La dispersión de su legado dificulta aún más este rompecabezas. En este sentido es donde las fuentes secundarias, como son cartas, prensa o ediciones como las tratadas en este artículo, pueden arrojarnos luz sobre tantas sombras en torno a su figura y sus actividades. Por poner un ejemplo de la magnitud de esta empresa, citaremos los trabajos de la musicóloga Matilde Olarte sobre su epistolario, estas cartas se encuentran dispersas entre ocho fondos, en muchas ocasiones sin catalogar. El enorme interés de este personaje para la cultura musical española de comienzos del siglo XX y su recepción en la costa este americana bien merecen el esfuerzo. La figura de Kurt Schindler se impone como punto clave de enlace entre la intelectualidad musical de la Segunda República y los aficionados americanos en torno a la Hispanic Society of America y a las actividades promovidas por Federico de Onís desde la Universidad de Columbia. 
Los apuntes biográficos aportados a continuación se basan en los estudios del musicólogo americano Israel J. Katz, musicólogo judío que ha realizado numerosos estudios sobre Kurt Schindler. Dichos apuntes se enriquecen con el currículum vitae que Kurt escribió con dieciocho años para su solicitud de ingreso a la Universidad. Como señalamos anteriormente, esta biografía necesita ser revisada y completada con un estudio en profundidad de las fuentes secundarias mencionadas. Sirvan estos apuntes como introducción al autor de la edición tratada en este artículo.

Nace en Berlín en 1882, en el entorno de una familia judía. Su padre era banquero y dueño de un sanatorio. Tiene un hermano menor, Ewald. En Berlín comienza su formación musical estudiando piano con Friedrich Gernsheim y composición con Ludwig Bussler, continuando posteriormente los de piano con Leopold Wolf y Conrad Ansorge. Muestra una gran precocidad con la lectura y la música, pudiendo ser considerado un niño prodigio. Una difteria severa y la escarlatina entre los 4 y los 6 años retienen al pequeño Kurt recluido en casa, donde sus padres le procuran una formación atendiendo a las inquietudes del niño, con profesores particulares y abundantes lecturas. Desde los siete años comienza a componer y desde los diez él ya reconoce su propósito firme de ser compositor. Con trece años volverá a resentirse su salud y abandona de nuevo la escuela durante un año. Durante esta etapa viaja en los veranos con sus padres por Alemania y Suiza como parte de su recuperación. Marie, su madre, se volcará en sus cuidados y en su educación, lo que contribuirá a crear un fuerte vínculo entre ellos.

Posteriormente, entre los años 1899 y 1901, continúa sus estudios en las Universidades de Berlín y de Múnich. Los de Musicología con Carl Stumpf y con Max Friedländer. En estos años de formación establece sus primeros contactos con la música folclórica, en particular a través de su maestro Friedländer y de los arreglos que escucha del repertorio popular en la Sociedad coral de Gernsheim. En estos años compone piezas de gran formato.

En 1902 presenta composiciones suyas en el Festival de Música de Krenfeld, donde figura junto a autores como Gustav Mahler y Richard Strauss. En el mismo año trabaja como director en la Ópera de Stuttgart, al año siguiente se hará cargo de la de Würzburg. Mottl y Strauss le ofrecen en 1904 un puesto como asistente en la Ópera de Berlín. En 1905 recibe una oferta de la Metropolitan Opera House de Nueva York por Heinrich Conried (1855-1909), para formar parte del equipo de dirección. Una gran desgracia familiar con origen en la quiebra del banco de su padre le lleva a aceptar esta propuesta y trasladarse a Estados Unidos. Antes pasa por París, donde hay noticia de su estancia allí en 1905. Desde 1907 trabaja para la editorial Gustav Schirmer como corrector, crítico y editor.

Ya instalado en Nueva York, crea el Coro MacDowell en 1909. Este coro se convertirá en 1912 en la Schola Cantorum de Nueva York, coro de gran renombre que colaborará con la Orquesta Filarmónica de Nueva York entre otros eventos. 
Schindler programa mucho repertorio basado en el folclore español y ruso, además de repertorio europeo medieval. Su amistad y su colaboración con la etnomusicóloga americana Natalie Curtis (1875-1921) influirían en sus estudios sobre la música popular. Como consecuencia de su interés y estudio de la cultura española, será nombrado miembro de la Hispanic Society of America, fundada por Mister Archer Huntington en 1904. Otro foco de interés de Schindler será el repertorio sefardí, como se observa en su catálogo. Este vínculo de la cultura judía con España pudo ser el origen del hispanismo de nuestro protagonista.

Entre 1912 y 1925 es el director coral y organista del Templo Enmanuel, con los que realizará giras por Europa. Schindler realiza trabajos de campo en Odessa, allí conoce a la que será su mujer, la actriz Vera Androuchevitch. Se casan en 1916, pero ella muere en 1918.

Su primer viaje a España documentado es en 1919, a la vuelta de una visita a Odessa. Constan sus visitas a Lluís Millet, director del Orfeón Catalán, y sus encuentros con otras personalidades de la música española como Arbós, Falla, Nin y Torner. En sus tres meses de estancia en nuestro país, recorrió gran parte del país. En otra visita a España en 1922 sería nombrado miembro honorario de la Fiesta de la Música Catalana, concurso de composición musical celebrado entre 1904 y 1922, promovido por el Orfeón Catalán.

De vuelta a Nueva York, se sabe que en 1926 trabajó un tiempo en el Teatro Roxy y en el Fórum Musical del Teatro Guild. Ese mismo año había dimitido como director de la Schola Cantorum.

En otoño de 1928 se encuentra de nuevo en España, esta vez con la intención de establecerse en Madrid y profundizar en su estudio de la cultura y la música de nuestro país. En 1929 organizó festivales en distintas capitales españolas como actividades para turistas de América y de países europeos. Eventos organizados para el Patronato Nacional de Turismo.

En los años 1929, 1930, 1932 y 1933 dedica largas estancias en España a la recogida sistemática de repertorio popular bajo el encargo de la Universidad de Columbia. En sus visitas recorre diversas regiones de nuestro país y en alguna ocasión comparte trabajos de campo con Torner y con el mismo Federico de Onís, que es su mentor en la Universidad de Columbia. Del material recogido, más de 1000 canciones, se nutre Folk Music and Poetry of Spain and Portugal, cancionero que publicaría la Universidad Columbia en 1941.

Entre sus dos últimas estancias en España, en 1931, Schindler asumió la dirección del recién creado Departamento de Música del Bennington College, en Vermont. Resultó ser una ocupación muy exigente y quizás contribuyó a empeorar su estado de salud. Hay constancia del avance de su enfermedad en su estancia de 1933 en España. Schindler moriría en 1935 en Nueva York. 
C. RAMÍREZ GARCÍA; Y. ALONSO VICARIO Y D. A. GÁLVEZ MÉNDEZ

CHANT DE TROUVĖRE, DE KURT SCHINDLER: ESTRENO Y PUBLICACIÓN EN NUEVA YORK

\section{ANÁLISIS DE LA OBRA}

Como ya se había mencionado con el Coro MacDowell, Kurt Schindler realizó una importante labor de rescate y divulgación de música medieval y folklórica rusa y española. Tal es el caso de Chant de Trouvère, estrenada en 1910 en el Madrigal Concert, un año antes de su publicación, por la editorial G. Schirmer. El hecho de que la canción fuera publicada a solo un año de su estreno en concierto pudiera ser indicio del éxito que tuvo en su momento, hecho que le valió para que la editorial apostara por su publicación tanto para el formato original (voz y dos arpas), como para voz y piano (edición sobre la que se basa el presente trabajo).

Kurt Schindler se había interesado en las investigaciones sobre música medieval realizadas por el musicólogo francés Pierre Aubry. Probablemente ese fue el modo en que conoció Kalenda Maya, una de las canciones más populares del siglo XII, que dio origen al arreglo realizado por Schindler. La autoría de esta canción se le atribuye al trovador Raimbaut de Vaqueiras, de quien se desconoce su fecha de nacimiento, pero se tienen noticias de su actividad musical desde 1180 hasta el 1207, año de su fallecimiento. Aun cuando Raimbaut de Vaqueiras nació en Francia, desarrolló gran parte de su vida en Italia, en la corte de Bonifacio de Montferrato, donde llegó a ser caballero.

En la edición de la Schirmer se ofrece una pequeña biografía de este autor, aunque no se revelan las fuentes de donde se extrajo dicha información. Todo parece indicar que este trovador gozó de fama durante su vida y que compuso una gran cantidad de canciones, aunque solo se conserva una edición de sus obras compuesta de treinta y tres canciones en donde aparece Kalenda Maya, que se encuentra actualmente en la Biblioteca Nacional de Francia.

La canción está basada en las festividades celebradas en algunas regiones europeas como Gales y Bretaña para festejar la llegada del verano. En la partitura se ofrece la letra en lengua provenzal, la traducción al francés realizada por M. Jeanroy y la versificación moderna realizada por Kurt Schindler. Estos textos se encuentran en la primera página de la partitura junto a la información biográfica de Raimbaut de Vaqueiras. En la parte en la que se ofrece la música aparece la versificación realizada por Schindler y la traducción del texto al inglés. Este último dato queda justificado por la tipología de público al que iba dirigida esta publicación, a saber, la burguesía neoyorkina de la época, que, aunque podían conocer la lengua francesa, era mejor proporcionarles la letra en su lengua materna.

El carácter doméstico de esta edición condiciona quizás las características musicales de la obra. Aun cuando la canción original era una melodía modal renacentista, Kurt Schindler realizó la tonalización de la misma. 
En el caso de la partitura que se analiza, la tonalidad es Mi Mayor, aunque en la portada se indica que para las voces de tenor o soprano es posible adquirir la partitura en Sol Mayor. Esta era una práctica habitual realizada por las casas editoriales de música para facilitar las partituras en las tonalidades adecuadas de cada tesitura y así hacerlas accesibles a cualquier pianista amateur.

\section{CHANSON DE TROUVERE}
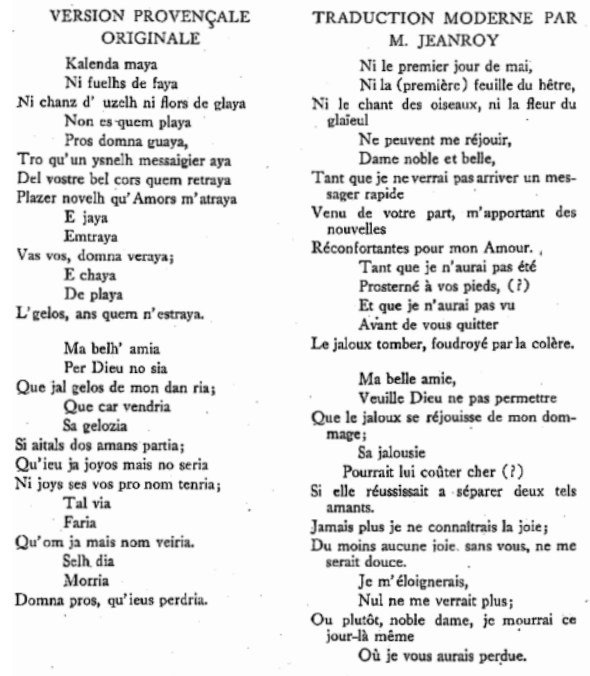

VERSIFICATION MODERNI

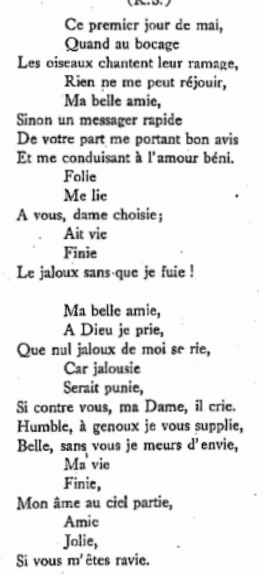

Texto en versión original, traducción moderna y versificación moderna.

El acompañamiento apoya la estructura de estrofa y estribillo del texto. En este caso solo se incluyen dos estrofas y el estribillo que no se interpreta de igual manera las dos veces que se repite, quedando así una forma musical binaria reflejada en el siguiente esquema:

A (la estrofa comienza con la frase Ce premier jour de mai y concluye con l'amour béni en el compás catorce)

B (estribillo, comienza con la frase Folie Me lie en el compás quince y termina con la frase sans que je fui en el compás veinticuatro)

A (la estrofa comienza con la frase Ma bell'amie en el compás veintiséis y termina con je meurs d'envie en el compás treinta y ocho)

B (estribillo, comienza con la frase Ma vie en el compás treinta y ocho y termina con la palabra ravie en el compás cuarenta y siete) 
La tonalidad de Mi Mayor se mantiene durante toda la obra. Schindler le da protagonismo a la relación tónica-subdominante en la armonización, de esta manera la canción conserva un poco de su carácter modal original. La cadencia perfecta aparece solo en dos ocasiones en la obra al final de cada estribillo para acentuar el carácter conclusivo de dichas partes. El primer ejemplo muestra las últimas frases de la primera vez que aparece el estribillo, la cadencia perfecta se encuentra entre el cuarto y quinto compás.

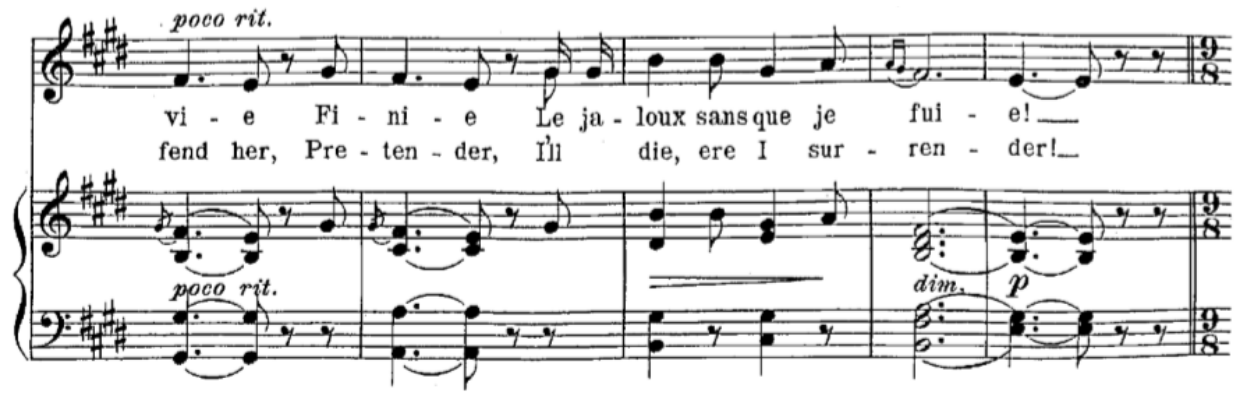

El segundo ejemplo representa el final de la obra y en este caso la cadencia se encuentra entre el tercero y cuarto compás.

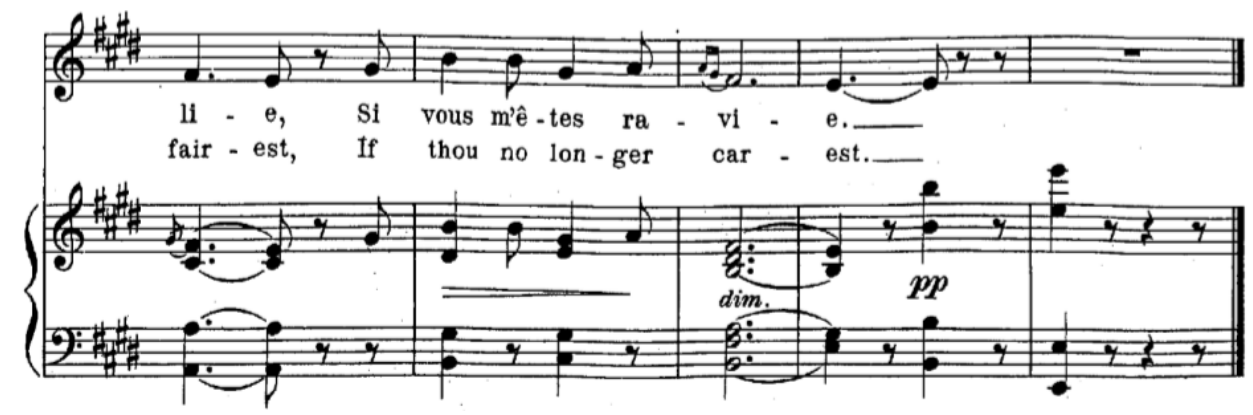

La obra se encuentra en tiempo ternario propio de los géneros danzables alternando entre $9 / 8$ y $6 / 8$. Dichos cambios coinciden con la aparición de la estrofa y el estribillo: estrofa 9/8; estribillo 6/8; estrofa 9/8; estribillo 6/8.

El acompañamiento del piano es sencillo, facilitando así la interpretación de la pieza a personas no profesionales. La obra comienza con dos compases introductorios realizados por el piano en los que se expone la relación tónica-subdominante que va a estar presente a lo largo de toda la obra. En el momento en que entra la voz interpretando la primera estrofa la mano derecha del acompañamiento pianístico dobla la melodía del cantante, esta era una práctica habitual en este tipo de arreglos

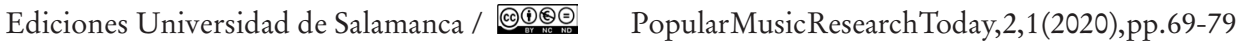


C. RAMÍREZ GARCÍA; Y. ALONSO VICARIO Y D. A. GÁLVEZ MÉNDEZ

CHANT DE TROUVĖRE, DE KURT SCHINDLER: ESTRENO Y PUBLICACIÓN EN NUEVA YORK

musicales. Del compás once al catorce se produce una imitación contrapuntística entre la voz y el piano, comportamiento que dinamiza y enriquece el discurso musical. Aun cuando se trata de una edición para uso doméstico, Kurt Schindler fue cuidadoso para adaptar el lenguaje musical al público que interpretaría la obra, pero manteniendo una lógica y coherencia estructural en la pieza.

\section{CONTEXTO DEL CONCIERTO DE ESTRENO}

El estreno de la obra Chant de Trouvère se hizo en el Concierto Madrigal organizado por Kurt Schindler, fue el 20 de marzo de 1910 en el hotel Waldorf-Astoria de Nueva York. Una nota de prensa del periódico The New York Times con fecha de 20 de marzo de 1910 anota que entre los suscriptores y titulares se encontraban destacadas señoras de la alta sociedad neoyorkina.

El periódico destaca la participación de la señora de Benjamin Lathrop y de Mlle. Jeanne Maubourg, que asistirían al Sr. Schindler en el canto durante el concierto. Así como de otras artistas como las señoritas Alma Glück, Janet Spencer, Edna Showalter, Ellen Learned y Mesara, de la Metropolitan Opera House. Los artistas de la Manhattan Opera Company eran Charles Gillbert, David Devries, Reinhold von Warlich, Berrick von Norden and Gustav Borde. El concierto se completaba con los solos de la señorita Glück y del señor Gillbert y cerraba con la recitación del prólogo una Oda a Palestrina de Victor Hugo, por Madame Mazarin. Era bastante usual que se informara en The New York Times de todos los conciertos que hacía Kurt Schindler, ya que el periódico era propiedad de una familia judía de Nueva York y se sabe que estos eventos y muchos más los organizaban judíos pertenecientes a la selecta élite de esta ciudad.

\section{LAS REDES}

En este apartado se intentará rastrear y dar nombre a la mayoría de estas distinguidas mujeres que fueron las filántropas, mecenas y el pilar fundamental de la gran red de contactos en la que Kurt Schindler se movía para financiar su trabajo, debido a su estatus social y exquisita educación. Además, hay que tener en cuenta que el Coro MacDowell lo conformaban estas señoras de alta sociedad. De hecho, las publicaciones que hacía con la editorial Schirmer eran para este público selecto que esperaban con ansia las nuevas creaciones de su director, cada concierto suponía un éxito rotundo y una nueva oportunidad de ventas para la editorial.

Schindler componía música para representarla en las obras de teatro de la Neighborhood Playhouse School of the Theatre, perteneciente a las hermanas Irene y Alice Lewisohn. Esta obra, Chant de Trouvère, también conocida como Kalenda Maya, se encuentra en el archivo de música incidental de esta escuela de teatro. 
La primera mujer que menciona la nota de prensa es la señora de Benjamin Lathrop, una de las asistentes del coro del director, que en realidad se llamaba Isabel Stevens Lathrop. Fue condecorada como miembro de la Legión francesa de Honor por su trabajo en París como presidenta del Fondo Americano para heridos franceses durante la Primera Guerra Mundial. El premio también fue por su importante papel en la fundación del American Memorial Hospital en Reims, Francia.

Entre los suscriptores y patrocinadores se encuentra la señora de John Innes Kane, descendiente de la multimillonaria familia Astor y dueños del hotel Waldorf-Astoria, ella se llamaba Annie Cottenet Schermerhorn. Quizás este vínculo pudiera haber sido la razón para que el concierto se realizara en ese lugar.

También se menciona a la señora de William H. Draper, ella era Mary Emma Carey. A la señora de William $\mathrm{H}$. Bliss se la puede encontrar como miembro de los 400 de Nueva York, su nombre era Anna Banes Bliss (nacida Anna Dorinda Blaksley), su marido llegó a ser director del Metropolitan Museum of Art.

Otro nombre es el de Therese Loeb, quien es mencionada en la nota de prensa como la señora de Jacob Schiff, un filántropo y poderoso banquero judío, nacido en Alemania y posteriormente nacionalizado norteamericano. Socio del banco de inversiones Kuhn, Loeb and Co.

La señora de Otto H. Kahn era Addie Wolff Kahn. Él también era socio del banco de inversiones Kuhn, Loeb and Co., juntos formaban un matrimonio reconocido por su papel de mecenas de las artes, coleccionistas y filántropos. Seguido podemos encontrar a la señora de Frederick Edey, Sarah Birdsall Otis. Fue una líder activista del movimiento para el sufragio de las mujeres y presidenta de la organización Girl Scouts of America.

Otra de las destacadas señoras era Julia Shipman, mujer del pastor Herbert Shipman. Era conocida por organizar grandes conciertos y veladas en homenaje a personajes políticos del momento. Se menciona también a la señora del general Cauncey McKeever, su nombre era Fanny, pero no se ha encontrado más información sobre ella.

El artículo continúa y nombra a la señora del coleccionista y abogado Howard Mansfield, cuyo nombre era Hellen Coolidge Tuttle, que fue miembro de la MacDowell Memorial Association y miembro de la junta directiva del MacDowell Club, entre muchos otros cargos en importantes asociaciones políticas, escuelas de música, movimiento de mujeres sufragistas, etc.

Se menciona a esta otra suscriptora, la señora de William Douglas Sloane, cuyo nombre era Emily Thorn Vanderbilt, filántropa y miembro de la prominente familia Vanderbilt. Ella financió la creación del Sloane Hospital for Women de Nueva York, actualmente forma parte del New York - Presbyterian Hospital.

A la que se refieren como la señora de James Speyer era en realidad Ellin Prince Lowery, fue una filántropa y dedicó gran parte de su tiempo y recursos 
en caridades para hospitales, ayudando a fundar el New York Skin and Cancer Hospital entre muchos otros organismos asociados a este campo. El matrimonio realizó donaciones significantes al Teachers College de la Universidad de Columbia.

La señora de Frederick Vanderbilt era Louise Holmes Anthony Vanderbilt, una filántropa preocupada por las oportunidades educacionales y de ocio para su comunidad local, Hyde Park, Nueva York. Una más de la familia Vanderbilt era la señora de Elliot Shepard. Su nombre era Margaret Louisa Vanderbilt Shepard, y como era tradición familiar fue filántropa y una apasionada defensora de la YWCA (Young Women's Christian Association), mandó construir un hotel especialmente para huéspedes transitorios de la YWCA.

De las siguientes mujeres se tiene muy poca o ninguna información, lo que indica que se debe seguir investigando más sobre este tema. La señora Gallatin era Ida Robinson Adams Gallatin, estuvo casada con James N., mejor conocido como Jack Gallatin; se sabe poco de su vida, aparte de su sonado divorcio. Y de la señora Riggs, solo se ha podido encontrar que era la hija del mayor Clark Reynolds. Hasta el momento no se tienen datos de la señorita Mary P. Field, la señora Charles F. Miller, la señora de Paul Morton y la señora de Emil Boas.

\section{CONCLUSIONES}

El estudio del Chant de Trouvère nos ha servido para analizar el contexto en el que tuvo lugar su edición. El público norteamericano al que iba destinada se impone como el principal condicionante de sus características. Junto a ello, el interés teñido de romanticismo por esa antigua Europa se manifiesta en distintos aspectos de la vida cultural norteamericana, como son los estudios en las universidades y la difusión de esta cultura pintoresca en exposiciones, conciertos, ediciones y representaciones teatrales. Kurt Schindler, por su sólida formación musical así como la amplitud de su cultura musical, puede acometer esta edición. Su integración en la élite de la sociedad neoyorquina por medio del Coro MacDowell y la relación ya establecida desde 1907 con la editorial G. Schirmer dan cuenta del marco que en pocos años había conseguido Kurt en esta nueva etapa americana. Ambas circunstancias hacen posible la edición y difusión de la partitura analizada en este artículo.

\section{BIBLIOGRAFÍA}

Frontera Zunzunegui, M. E. (2014). Una figura en el campo de la música tradicional española en el primer tercio del siglo XX. El polifacético músico alemán Kurt Schindler (1882-1935). (Tesis doctoral). Salamanca: Universidad de Salamanca.

Katz, Israel J. (1999-2002). Schindler, Kurt. En E. Casares Rodicio (Coord.), Diccionario de la Música Española e Hispanoamericana, vol. 9 (pp. 860-861). Madrid: Sociedad General de Autores y Editores de España. 
C. RAMÍREZ GARCÍA; Y. ALONSO VICARIO Y D. A. GÁLVEZ MÉNDEZ

CHANT DE TROUVĖRE, DE KURT SCHINDLER: ESTRENO Y PUBLICACIÓN EN NUEVA YORK

Olarte Martínez, M. (2011). La correspondencia inédita de Kurt Schindler como una fuente directa para contextualizar la vida musical del primer tercio del siglo XX. En M. Olarte Martínez (Ed. y Coord.), Fuentes documentales interdisciplinares para el estudio del patrimonio y la oralidad en España (pp. 554-630). Bayona: Editorial Dos acordes.

Schindler, K. (1911). Chant de Trouvère (Troubadour Song). New York: G. Schirmer.

Steinhauer, J. (2013). Chronologisches Verzeichnis der Kompositionen und Volksliedbearbeitungen von Kurt Schindler. En M. Olarte Martínez y P. Capdepón Verdú (Eds.), La música acallada: Liber Amicorum José García Laborda (pp. 313-333). Salamanca: Editorial Amaru.

\section{Fuentes electrónicas}

Catálogo de la Biblioteca Nacional de Francia. Recuperado el 03 de 02 de 2020, de https:// catalogue.bnf.fr/ark:/12148/cb44831488m.

Dumbarton Oaks. (s. f.). Recuperado el 25 de 04 de 2019, de https://www.doaks.org/ resources/bliss-tyler-correspondence/annotations/william-henry-bliss.

Dumbarton Oaks. (s. f.). Recuperado el 25 de 04 de 2019, de https://www.doaks.org/ resources/bliss-tyler-correspondence/annotations/anna-barnes-bliss.

Find a Grave. (s. f.). Recuperado el 24 de 04 de 2019, de https://www.findagrave.com/ memorial/170812298/fanny-mckeever.

Find a Grave. (s. f.). Recuperado el 25 de 04 de 2019, de https://www.findagrave.com/ memorial/67276221/sarah-birdsall-edey.

Getty Images. (s. f.). Recuperado el 25 de 04 de 2019, de https://www.gettyimages.es/ detail/v\% C3\% ADdeo/mrs-herbert-shipman-makes-opening-remarks-as-she-v\%C3\%ADdeos-de-noticias/579050528.

Grove Music Online. (2001). Recuperado el 22 de 04 de 2019, de https://doi.org/10.1093/ gmo/9781561592630.article.24872.

The Frick Collection. (s. f.). Recuperado el 24 de 04 de 2019, de http://research.frick.org/ directoryweb/browserecord.php?-action=browse\&-recid $=6770$.

The New York Times. (s. f.). Recuperado el 24 de 04 de 2019, de https://www.nytimes. com/1908/05/31/archives/americans-share-court-ball-honors-also-prominent-atlondon-gala.html.

The New York Times. (s. f.). Recuperado el 25 de 04 de 2019, de https://www.nytimes. com/1964/10/28/mrs-benjamin-lathrop-dies.html.

www.immigrantentrepreneurship.org. (s. f.). Recuperado el 25 de 04 de 2019, de https:// www.immigrantentrepreneurship.org/entry.php?rec=222.

www.newspapers.com. (s. f.). Recuperado el 21 de 04 de 2019, de https:// img.newspapers.com/img/img ? institution $\mathrm{Id}=0 \& \mathrm{us}$ er $=0 \& \mathrm{i}$ $\mathrm{d}=408818178 \&$ width $=557 \&$ height $=1208 \& \mathrm{crop}=2415 \_1012 \_1418 \_3134$ \&rotation $=0 \&$ brightness $=0 \&$ contrast $=0 \&$ invert $=0 \& \mathrm{ts}=1539829002 \& \mathrm{~h}=\mathrm{e} 867 \mathrm{f3} \mathrm{c} 127 \mathrm{e}-$ 436ba42c7ab958358b9e5. 
